\title{
Perspectivas da utilização de resíduos urbanos lignocelulósicos para confecção de painéis
}

\author{
Nayara Guetten Ribaski ${ }^{1}$, Ugo Leandro Belini ${ }^{2}$ \\ ${ }^{1}$ UFPR e PUCPR, doutoranda PPGSAU, nayribaski@hotmail.com \\ 2 UTFPR - PPGSAU, pós-doutor, ubelini@utfpr.edu.br
}

\begin{abstract}
RESUMO: Há crescente demanda de instituições de pesquisa para promover estudos com matérias primas consideradas resíduos e oriundas de recursos lignocelulósicos, promovendo novos usos e aplicações bem como agregando valor aos materiais. Neste contexto, o presente trabalho aborda aspectos envolvidos na disponibilidade e características dos produtos resultantes da poda de árvores urbanas, bem como detalha etapas e tecnologias envolvidas na utilização de resíduos desta poda urbana para produção de painéis reconstituídos de partículas, produto este de ampla aceitação e aplicação nas diversas áreas da Bioengenharia. As questões que que norteiam o estudo é a verificação de disponibilidade, e tecnologia conhecida, para uso de resíduos de poda de árvores urbanas visando transformação em painéis reconstituídos. Tentar agregar valor a este recurso lignocelulósico e mitigar disposições incorretas deste material. E verificar o tamanho das partículas finais visando adequações à qualidade de produto e uso final, bem como utilizar aglutinantes biodegradáveis ou com baixa emissão de formaldeído.
\end{abstract}

PALAVRAS-CHAVE: Resíduos, arborização, sustentabilidade

\begin{abstract}
There is a growing demand from research institutions to promote studies with raw materials considered waste and from lignocellulosic resources, promoting new uses and applications as well as adding value to the materials. In this context, the present work addresses aspects involved in the availability and characteristics of products resulting from the pruning of urban trees, as well as details stages and technologies involved in the use of residues from this urban pruning to produce reconstituted particle boards, a product that is widely accepted and application in different areas of Bioengineering. The questions that guide the study are the availability check, and known technology, for the use of urban tree pruning residues aiming at transformation into reconstituted panels. Trying adds value to this lignocellulosic resource and mitigates incorrect dispositions of this material. And check the size of the final particles in order to adapt them to the quality of the product and end use, as well as to use biodegradable binders or those with a low formaldehyde emission.
\end{abstract}

KEYWORDS: Waste, afforestation, sustainability.

\section{INTRODUÇÃO}

As cidades brasileiras apresentam, em seu panorama urbano, uma quantidade relativamente significativa de árvores, em geral plantadas não somente com objetivos estéticos, mas também para amenizar as altas temperaturas que ocorrem no verão, em grande parte da área territorial habitada da nação (CHAHUD et al., 2013).

Porém, a quantidade de resíduos gerada pela atividade da poda dos galhos das referidas árvores pode alcançar um volume expressivo. No tocante à cidade de Curitiba, a coleta de resíduos vegetais (podas da arborização pública, limpeza de jardins), equivale a 1.770 ton/mês (PREFEITURA DE CURITIBA, 2011) e apresenta um riquíssimo material para confecção de produtos de maior valor mitigando, ainda, possível disposição incorreta.

Os resíduos de podas são os resíduos gerados das atividades de manejo da arborização urbana e também podem ser classificados como riscos potenciais ao meio ambiente.

Neste contexto, a crescente consciência da sociedade com a necessidade de preservação do meio ambiente, bem como a demanda de produtos ecologicamente corretos, resultou na busca por materiais de fontes renováveis envolvendo setores econômicos, sociais e industriais, atrelando estes aspectos ao conceito de Desenvolvimento Sustentável. Segundo WWF-Brasil, para que este desenvolvimento ocorra é necessário qualidade em vez de quantidade, reduzindo o uso de matériasprimas e produtos com aumento da reutilização e reciclagem.

Os RSU, de acordo com a norma NBR.10.004 (ABNT, 2004), são resultantes da atividade doméstica e comercial dos centros urbanos, caracterizados como os que não podem ser descartados em rede pública de esgotos nem mesmo em corpos de água (RIBASKI \& realização organized by apoio institucional supported by
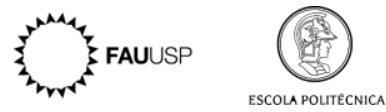
BELINI, 2019). E a mesma norma menciona ainda os critérios de riscos potenciais ao meio ambiente, onde os resíduos de podas ou arborização urbana, apesar do alto teor de lignina, são biodegradáveis e classificados como Classe II-A não inertes.

Os resíduos de poda e remoção da arborização urbana geram resíduos na forma de galhos, ramos, folhas, sementes, frutos, fustes e até mesmo raízes são considerados resíduos verdes (LIMA, 2004; MEIRA, 2010; CHALUPPE, 2013).

Esses redíduos possuem um grande potencial de utilização, pois são abundantes e independem do mercado econômico justamente por não depender de uma atividade industrial. Porém a dificuldade na utilização dos resíduos de poda urbana reside, na maior parte das vezes, na gestão amadora e desorganizada feita pelos municípios brasileiros, que geralmente não têm controle da quantidade gerada e acabam desperdiçando este importante insumo (CORTEZ, 2011, p.44; VALE, 2016).

Destaca-se que este material não recebe um tratamento e aproveitamento adequado. No entanto, os resíduos de madeira gerados que não são utilizados podem deixar de ser um passivo ambiental, sendo processados como matéria-prima para diversos fins, incluindo a fabricação de painéis, podendo gerar lucro para a iniciativa privada e reduzir problemas ambientais de interesse da sociedade (RIBASKI \& BELINI, 2019).

A valorização de resíduos sólidos pode ser compreendida como a reutilização destes resíduos, de modo que não seja a destinação final em aterros sanitários (BIDONE, 2001). O aproveitamento do resíduos verdes é uma excelente opção para agregar valor a novos produtos, e segue tendência de pesquisas referente ao aproveitamento de outros recursos lignocelulósicos, considerados como resíduos, para a confecção de painéis reconstituídos (BELINI et al, 2012; OLIVEIRA et al, 2016; FIORELLI et al 2017).

Segundo Chaluppe (2013), as formas de valorização dos resíduos de podas podem ser divididas em duas linhas: sem aproveitamento energético e com aproveitamento energético.

Em relação ao aproveitamento não energético, podem-se citar a reutilização pela confecção de novos compósitos e a reciclagem orgânica através dos processos de compostagem e vermicompostagem (CORTEZ, 2011).

No aproveitamento energético, estão compreendidas as tecnologias relacionadas a processos físicos (briquetagem e peletização), os processos termoquímicos (combustão, pirólise e gaseificação), além dos processos biológicos (digestão anaeróbica) (CHALUPPE, 2013).

Ainda, o setor de resíduos da arborização urbana é gerenciado inadequadamente, principalmente os gerados pela poda de árvores e pela manutenção de jardins. A falta de gestão dos resíduos da arborização urbana tem contribuído para agravar os problemas ambientais, sociais, econômicos resultantes da disposição inadequada desses materiais (MEIRA, 2010). Iniciativas como a solução de um material ecológico provindo destes resíduos pode gerar um faturamento para a prefeitura, redução de custos de manutenção de acúmulo de material orgânico em aterros sanitários e novos empregos.

O foco desse trabalho são os resíduos de madeira oriundo das podas de arborização urbana público/privada, sem aproveitamento energético, para transformação em painéis reconstituídos.

\section{DESENVOLVIMENTO}

\subsection{Matéria Prima}

Os resíduos de poda, por serem constituídos por uma diversa gama de materiais e pelo fato da produção de determinados materiais estar sujeita ao fator sazonalidade, são heterogéneos produzindo amostras igualmente heterogéneas, o que dificulta a sua caracterização (PEREIRA, 2013, p.5).

As espécies arbóreas apresentam grande variabilidade na composição de sua estrutura interna, o que concerne na diversidade dos elementos anatômicos da madeira, como propriedades físicas, químicas e mecânicas. A descrição anatômica de madeiras, especificamente, é de fundamental importância para a determinação de seu aproveitamento tecnológico. Segundo, Oliveira et al. (2006), está baseado no fato de que as dimensões, a frequência e o arranjo dos elementos anatômicos têm grande influência sobre as propriedades físicas e mecânicas da madeira. Esses são parâmetros que visam estabelecer o potencial das diferentes espécies para as mais variadas utilizações (TRIANOSKI, 2012).

Ao utilizar resíduos oriundos de podas urbanas, pode não ser possível realizar a segregação ou até mesmo a identificação das espécies arbóreas que compõem aquela amostra coletada, visto heterogeneidade de gêneros e espécies florestais que a compõem.

Visando sua utilização no processo produtivo de painéis de madeira, inúmeras variáveis interagem influenciando as propriedades do produto final, sendo eles fatores inerentes à madeira e inerentes ao processo. As principais variáveis de influência e controle são a massa específica da madeira, densidade do painel, geometria das partículas, teor de umidade, tipo e teor do adesivo, método de formação do colchão e parâmetros de prensagem (MOSLEMI, 1974; KELLY, 1977; MALONEY 1993, BRITO et al., 2005; TRIANOSKI, 2010). Parâmetros estes que podem ser 
mensurados mesmo havendo um mix de espécies dentro de uma amostra.

A massa específica varia de uma espécie para outra, assim como dentro da mesma espécie e da mesma árvore, tanto no sentido base-topo como no sentido medula-casca (KOLLMANN, 1959; PANSHIN; DE ZEEUW, 1980; KLOCK, 2000).

Em termos de caracterização tecnológica, tanto de espécies desconhecidas quanto de espécies já estudadas a determinação da massa específica é fundamental devido sua influência na resistência mecânica e na estabilidade dimensional, sendo esta determinação e avaliação, um dos pontos chave para a confecção de painéis contendo uma diversidade de espécies arbóreas desconhecidas dentro de uma mesma amostra.

\subsection{Manufatura dos Painéis}

O fluxograma ilustrado na Figura 1 indica as principais etapas envolvidas na confecção de painéis reconstituídos em laboratório.

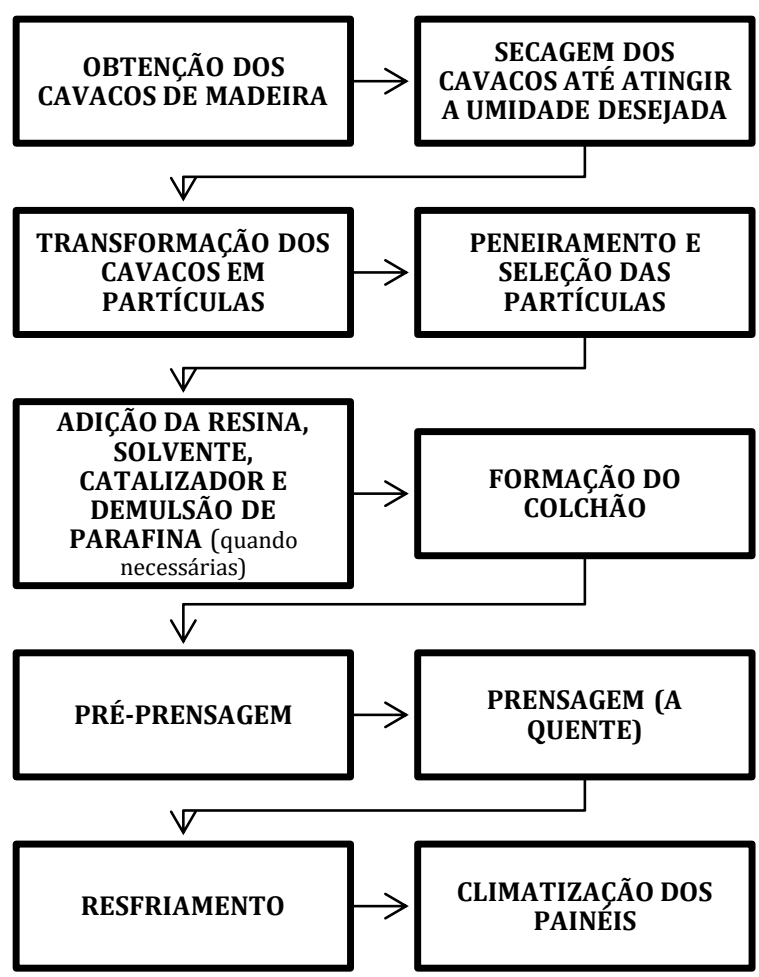

Figura 1: Imagem representando as etapas para a confecção dos painéis utilizando os resíduos de poda urbana.

Fonte: Adaptado de Iwakiri, 2005.

\subsection{Obtenção dos Cavacos de Madeira}

Na região de Curitiba, o resíduo da poda urbana é recolhida por algumas empresas licitadas pela prefeitura, e o material utilizado nessa pesquisa será fornecido pela empresa Byocom - Destinação de resíduos vegetais e de madeira, localizada em Fazenda Rio Grande-PR, conforme Figura 2.

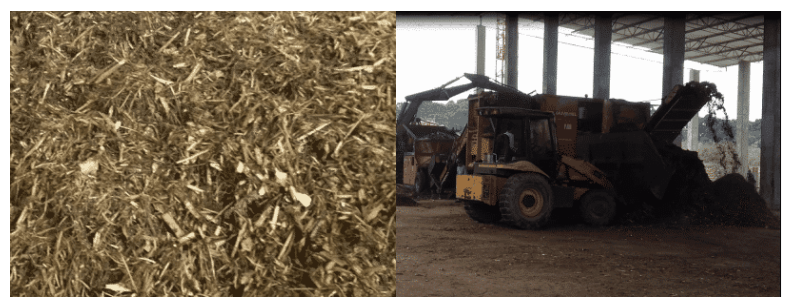

Figura 2 - Partículas geradas na empresa Byocom

O resíduo primário da poda pode ser processado em diversos tamanhos de partículas envolvendo, a princípio, granulometria entre $10 \mathrm{~mm}$ e $100 \mathrm{~mm}$ e umidade em torno de $30 \%$. Os tamanhos indicados permitem ampla flexibilidade na seleção e estabelecimento de condições de obtenção focadas no uso final dos painéis.

O material oriundo da Byocom não recebe nenhuma segregação com relação a identificação das espécies madeireiras antes de iniciar o processo de geração de partículas, ou seja, o material obtido será um grande mix de espécies.

\subsection{Secagem dos cavacos até atingir a umidade desejada}

O processo de secagem dos cavacos ocorrerá em duas etapas. A primeira etapa consisti na redução do teor de umidade inicial, sendo esta efetuada ao ar livre. A segunda etapa será conduzida em estufa convencional com circulação de ar forçada, em aparato específico para secagem de partículas, a temperatura de $100 \circ \mathrm{C}$, resultando num teor de umidade final de aproximadamente 3-6\%, dependendo do tipo de adesivo a ser utilizado, para que haja uma boa penetração.

O teor de umidade é encontrado a partir da porcentagem de água extraída de uma amostra, quando está é submetida a certa temperatura, até sua massa tornar-se constante (NBR 14.929-04/2017). Seu valor é definido em relação à massa seca, conforme a expressão:

$$
\mathrm{U}=((\mathrm{MU}-\mathrm{MS}) \div \mathrm{MS}) \times 100
$$

onde $U$ - Umidade residual do material, dada em porcentagem

$\mathrm{MU}$ - massa úmida do material em gramas

MS - massa seca em gramas.

Será utilizado o método gravimétrico ou da pesagem para a determinação do teor de umidade do ensaio, de acordo com a ABNT NBR 14.810-3 (Chapas de madeira aglomeradas Parte 3: Métodos de ensaio). 


\subsection{Transformação dos cavacos em partículas}

Após a secagem as mesmas serão conduzidas ao moinho martelo para redução de tamanho das partículas de madeira. Em seguida, esse material passará por peneiras manuais, sendo classificado de acordo com a sua granulometria.

\subsection{Peneiramento e seleção das partículas}

Os cavacos deverão passar na peneira de 40 mesh e ficar retidas na peneira de 60 mesh, conforme a Norma TAPPI 264:1997. Após a classificação será determinado as amostras para a confecção dos painéis e será separado uma quantidade suficiente de cavacos de forma a garantir representatividade de todo o material processado.

O material será armazenado em embalagens plásticas para evitar a absorção da umidade do ambiente.

\subsection{Adição de elemento aglutinante}

A aplicação da resina objetiva aglutinar as partículas e assim formar o painel. Moslemi (1974) afirma que após a aplicação do adesivo, normalmente em condição aquosa, o teor de umidade do colchão de partículas aumenta para um intervalo entre 8 e $14 \%$.

Ainda, esta etapa permite a pesquisa e uso de adesivos biodegradáveis ou com baixa emissão de formaldeído, classificados como produtos classe E1 em emissão.

\subsection{Formação do colchão}

Após a mistura das partículas com o adesivo, irá se formar uma massa, que será espalhada e orientada de forma aleatória dentro de uma caixa sem fundo (caixa formadora), com dimensões de $50 \times 50 \mathrm{~cm}$. Essa caixa serve para ajustar o produto inicial para que ele fique nos moldes para a prensagem.

\subsection{Pré-prensagem}

Uma importante etapa é a pré-prensagem, que pode ocorrer de forma manual para melhor acomodação das partículas da massa dentro do molde e para o ganho de coesão do colchão. Alguns trabalhos indicam a espera de 24 horas antes de ir para a prensagem hidráulica.

\subsection{Prensagem a quente}

Posteriormente, o colchão é colocado na prensa hidráulica com temperatura de prensagem de 90 으. $\mathrm{O}$ processo de prensagem dos compostos consistiu no emprego de uma pressão 3,5 MPa durante 3 minutos, seguido do alivio de pressão da prensa por um período de 30 segundos e posterior emprego da pressão de $3,5 \mathrm{MPa}$ durante $7 \mathrm{~min}$, totalizando tempo de prensagem de $10 \mathrm{~min}$ com 30 seg para liberação de gases.

\subsection{Climatização dos painéis}

Após a manufatura dos painéis, os mesmos serão conduzidos para a câmara de climatização com condições ambientais controladas $\left(20 \pm 3^{\circ} \mathrm{C}\right.$ e $65 \pm 5 \%$ UR), até atingirem a umidade de equilíbrio nestas condições.

\section{CONCLUSÕES}

- Há disponibilidade, e tecnologia conhecida, para uso de resíduos de poda de árvores urbanas visando transformação em painéis reconstituídos.

- Seu uso em painéis agrega valor a este recurso lignocelulósico e mitiga disposições incorretas deste material.

- É possível alterar tamanho das partículas finais visando adequações à qualidade de produto e uso final, bem como utilizar aglutinantes biodegradáveis ou com baixa emissão de formaldeído.

- Este artigo demonstra as principais etapas envolvidas na confecção de painéis reconstituídos em laboratório.

\section{AGRADECIMENTOS}

Ao Programa de Pós Graduação em Sustentabilidade Ambiental Urbana (PPGSAU) da UTFPR-Curitiba.

\section{REFERÊNCIAS}

1. ABNT (Associação Brasileira de Normas Técnicas) (2004). ABNT NBR 10004/2004. Resíduos Sólidos.

2. ABNT (Associação Brasileira de Normas Técnicas) (2006). NBR 14.810-3/2006. Chapas de madeira aglomeradas Parte 3: Métodos de ensaio

3. ABNT (Associação Brasileira de Normas Técnicas) (2017). NBR 14.929-04/2017. Madeira - Determinação do teor de umidade de cavacos - Método por secagem em estufa

4. BELINI, U. L.; TOMAZELLO FILHO, M.; LOUZADA, J. L. P. C.; RODRIGUES, J. C.; ASTOLPHI, J. R. S. (2012) Pilot study for MDF manufacture from sugarcane bagasse. 
Holz als Roh- und Werkstoff (Print) / European Journal of Wood and Wood Products (Print), v. 70, p. 537-539.

5. BRITO, E. O. et al. (2005). Chapas de madeira aglomerada de uma camada de Pinus elliottii Engelm com a adição das cascas de Eucalyptus pellita F. Muell. Cerne, v. 11, n. 4, p. 369-375.

6. CORTEZ, L. C. (2011). Estudo do potencial de utilização da biomassa resultante da poda de árvores urbanas para a geração de energia: Estudo de Caso: AES ELETROPAULO. São Paulo, 2010. 246p. Tese (Doutorado). Universidade de São Paulo, São Paulo,

7. CHALUPPE, M.A.C. (2013). Análise da Implantação do Projeto "Valorização dos Resíduos Sólidos Orgânicos no Município de Florianópolis Através do Beneficiamento dos Resíduos de Podas". Trabalho de conclusão de curso em Engenharia sanitária e ambiental, Universidade Federal de Santa Catarina.

8. FIORELLI, J.; GALO, R. G.; CASTRO JR., S.L.; BELINI, U. L.; LASSO, P. R. O.; SAVASTANO JR, H. (2017). Multilayer Particleboard Produced with Agroindustrial Waste and Amazonia Vegetable Fibres. Waste and Biomass Valorization, v. 8, p. 1-11.

9. IWAKIRI, S. (2005). Painéis de madeira reconstituída. Curitiba Fupef, $247 \mathrm{p}$.

10. KELLY, M. W. (1977). A Critical literature review of relationships between processing parameters and physical properties of particleboards. U.S. For. Prod. Lab. General Technical Report FPL-10, 66 p.

11. KLOCK, U. (2000). Qualidade da madeira de Pinus maximinoi H. E. Moore. 291 f. Tese (Doutorado em Ciências Florestais) - Setor de Ciências Agrárias, Universidade Federal do Paraná, Curitiba.

12. KOLLMANN, F.F.P.; COTÊ, W.A. (1968). Principles of wood science and technology. Berlim: Springuer Verlag, v. 1, 592 p.

13. MALONEY, T. M. (1993). Modern particleboard and dry-process fiberboard manufacturing. 2. ed. São Francisco: Miller Freeman, 689 p.

14. MEIRA, A.M. (2010). Gestão de resíduos da arborização urbana. 179p. Tese de Doutorado (Ciências: Recursos Florestais, com opção em Tecnologia de Produtos Florestais) - Universidade de São Paulo, Piracicaba - SP.

15. MOSLEMI, A. A. (1974). Particleboard. London: Southern Illinois University Press, 245 p.
16. OLIVEIRA, E.; VITAL, B.R.; PIMENTA, A.S.; DELLA LUCIA, R.M.; LADEIRA, A.M.; CARNEIRO, A.C.O. (2006). Estrutura anatômica da madeira e qualidade do carvão. Árvore, v. 30, n.2, p.311-318.

17. OlIVEIRA, R.F. MENDES, L. M. MENDES, T. P. FREIRE (2016). Particleboard Panels Made From Sugarcane Bagasse: characterization for use in the furniture industry, Mat. Res. 19 (2016) 914-922. https://doi.org/10.1590/1980-5373-MR-2015-0211.

18. PANSHIN, A.J.; DE ZEEW, C. (1980). Textbook of wood technology: II. New York, MC Graw Hill, 4 ed., $722 \mathrm{p}$.

19. PEREIRA, A.I.C. (2013). Co-digestão anaeróbia de resíduos verdes e lamas de ETAR para produção de biogás. 2013. P.98. Dissertação (Mestrado em Energia e Bioenergia) - Faculdade de Ciências e Tecnologia, Universidade Nova de Lisboa. Lisboa, Portugal.

20. RIBASKI, N. G.; BELINI, U. L. (2019). Aproveitamento de resíduos sólidos urbano madeireiro. Brazilian Journal of Tecnology. Vol 2, No 3, $742-757 p$.

21. TAPPI. Technical Association of the Pulp and Paper Industry Atlanta. Tappi test methods. T264 cm-97, Atlanta: TAPPI: press, 1997.

22. TRIANOSKI, R. (2010). Avaliação do potencial de espécies florestais alternativas, de rápido crescimento, para produção de painéis de madeira aglomerada 262 f. Dissertação (Mestrado em Engenharia Florestal - Setor de Ciências Agrárias, Universidade Federal do Paraná, Curitiba.

23. VALE, V.H.D. (2016). Diagnóstico dos resíduos de podas do município de Natal/RN. TCC (Engenheiro Ambiental). Universidade Federal do Rio Grande do Norte, Natal - RN.

24. ZOBEL, B. J.; THORBJORNSEN, E.; HENSON, F. (1960). Geographic, site and individual tree variation in wood properties of loblolly pine. Silvae Genetica, $n$. 9, p. 149-158. 